la revue La revue pour l'histoire du CNRS

POUR LHISTORE DU CNRS $\quad 24 \mid 2009$

Soixante-dixième anniversaire du CNRS

\title{
La difficile mise sur le marché de nouveaux antibiotiques
}

Jean-Paul Leonetti

\section{OpenEdition}

Journals

Édition électronique

URL : https://journals.openedition.org/histoire-cnrs/9103

DOI : 10.4000/histoire-cnrs.9103

ISSN : 1955-2408

Éditeur

CNRS Éditions

Édition imprimée

Date de publication : 5 octobre 2009

ISSN : 1298-9800

Référence électronique

Jean-Paul Leonetti, "La difficile mise sur le marché de nouveaux antibiotiques », La revue pour l'histoire du CNRS [En ligne], 24 | 2009, mis en ligne le 05 octobre 2009, consulté le 20 mai 2021. URL : http:// journals.openedition.org/histoire-cnrs/9103; DOI : https://doi.org/10.4000/histoire-cnrs.9103

Ce document a été généré automatiquement le 20 mai 2021.

Comité pour l'histoire du CNRS 


\title{
La difficile mise sur le marché de nouveaux antibiotiques
}

\author{
Jean-Paul Leonetti
} Bernard Lebleu à l'Institut de génétique moléculaire de Montpellier (IGMM). Puis, j'ai décidé de changer et de me diriger vers la science dure à visée non thérapeutique. J'ai rejoins le Center for Molecular Genetics de l'université de San Diego avec Peter Geiduschek pendant deux ans et demi.

en France, je souhaitais continuer dans le domaine de la bactériologie et ayant déjà travaillé sur la transcription bactérienne, je me suis intéressé à des aspects de la recherche plus ou moins appliqués. Je pensais alors que le domaine académique avait son rôle à jouer. Si l'on observe les domaines de recherche qui se développent à un moment donné, il s'agit de domaines convoités et portés par les industriels. Par exemple, la recherche sur le cancer est un secteur porteur donc soutenu par les industriels et l'État. En revanche, le domaine anti-infectieux «ne fait plus vraiment recette » et certains industriels ont revendu depuis longtemps leur département d'infectiologie. Aventis en fait partie. Pas assez rentable selon eux. Pour preuve, aucun nouvel antibiotique n'a été mis sur le marché entre 1960 et 1995. Les deux seules nouvelles molécules datent des années 2000, ce qui montre bien l'orientation prise par les industriels depuis les années 1960. En dehors du désintérêt des industriels, il y a un problème réglementaire. Pour qu'un médicament arrive sur le marché, on doit prouver qu'il est plus efficace que les autres molécules utilisées. Des molécules globalement moins efficaces mais plus complémentaires auraient un intérêt. Or, en infectiologie, on travaille sur des molécules qui touchent seulement $30 \%$ de la souche et qui viennent compléter la précédente molécule. Cela montre la limite des politiques industrielles et de santé publique. À mon retour des États-Unis, j'ai travaillé avec Bernard Pau ${ }^{1}$, un ancien de Sanofi, directeur de l'Institut de biotechnologie et pharmacologie à la faculté de Montpellier. J'ai monté une équipe avec laquelle je travaille encore aujourd'hui. À l'époque, il y avait peu de recherches en bactériologie. 

La revue... Pourquoi avoir tenté l'aventure de la création d'entreprise ?

J.-P. L. J'ai rencontré Olivier Thaler, microbiologiste à l'Emip-Inra ${ }^{2}$, initiateur du projet de création d'une société et The Duc Hua, biochimiste. Nous avons décidé de fonder Selectbiotics dans le but d'isoler et de caractériser de nouveaux antibiotiques d'origine bactérienne pour l'industrie pharmaceutique. Selectbiotics a vu le jour en avril 2001. L'objectif de nos recherches réciproques était de trouver de nouveaux antibiotiques, de les amener aux essais cliniques, puis de les céder à une entreprise plus importante. Pour pouvoir me greffer au projet de cette société, j'ai fait des demandes auprès du Comité d'éthique du CNRS.

La revue... Comment s'est déroulée la création de Selectbiotics? Et son développement?

7 J.-P. L. Grâce au soutien du CNRS, Selectbiotics a été primée deux années consécutives par l'Anvar et les ministères en charge de la recherche et de l'éducation. Elle a reçu deux prix, un pour le prémontage du projet et un second, mieux doté, pour le démarrage de la société. L'Association française pour la recherche thérapeutique lui a décerné le grand prix industriel. Le lancement a eu lieu parce qu'un investisseur était intéressé et nous incitait fortement à le faire. Malheureusement, le 11 septembre $2001 \mathrm{a}$ bouleversé la donne, en particulier tous les problèmes et fluctuations boursières qui en ont découlé. Dès lors, la plupart des investisseurs se sont momentanément retirés des domaines à risques comme le nôtre. Notre interlocuteur au CNRS était la cellule régionale de valorisation du CNRS qui dépend de la Direction de la politique industrielle (DPI). Le parcours a été semé d'embûches, car nous n'avons jamais pu faire le passage de la démonstration du laboratoire à une démonstration industrielle par manque de financements. Concernant Selectbiotics, nous n'avons pas trouvé le fonds d'amorçage qui aurait permis à notre projet de sortir du laboratoire. Aussi, Selectbiotics a pu survivre tant bien que mal durant trois ou quatre ans, grâce notamment au soutien de l'Anvar. Mais un brevet en dormance perd un peu de sa valeur. Pour passer à la vitesse supérieure, une société doit trouver rapidement des investisseurs publics ou privés.

8 La revue... Quel a été le soutien du CNRS ? Et, selon vous, quelles ont été les principales difficultés rencontrées?

9 J.-P. L. Grâce au CNRS, les activités de notre société ont été accueillies dans ses laboratoires et un salarié a été affecté pour la recherche dans chacun d'entre eux. La démarche entrepreunariale est bien spécifique et le chercheur n'y est pas préparé. Il ne sait pas ce que l'industriel attend de lui. La démarche scientifique pousse à la prudence, mais celle-ci n'est pas payante auprès d'un investisseur. Une fois l'aval du CNRS obtenu, le chercheur est un peu « lâché » dans la nature. Il n'a pas une idée précise du circuit qu'emprunte son dossier, n'a pas la connaissance des différents brevets exploitables ou non, de leurs aspects juridiques, des conditions dans lesquelles le CNRS peut les déposer... Il manque de préparation et de formation pour pouvoir «faire le grand saut ». Pour moi, la création d'entreprise reste tout de même le meilleur moyen pour le transfert de technologies vers l'industrie. Malgré tout, cette expérience reste enrichissante mais les parcours en dents de scie sont épuisants. Aujourd'hui, je ne regrette rien, mais mes compagnons d'" infortune » et moi-même ne sommes pas encore tout à fait prêts à nous relancer dans l'aventure... 


\section{NOTES}

1. Professeur d'immunologie et de biotechnologie à l'université de Montpellier I.

2. Écologie microbienne des insectes et interactions hôtes pathogènes-Institut national de la recherche agronomique.

\section{RÉSUMÉS}

Suite à une rencontre avec deux collègues chercheurs, Jean-Paul Leonetti, spécialiste du domaine anti-infectieux, a décidé de se greffer à un projet de création d'entreprise en 1999. Il en retrace ici toutes les étapes.

\section{AUTEUR}

\section{JEAN-PAUL LEONETTI}

Jean-Paul Leonetti est chargé de recherche au CNRS au Centre d'études d'agents pathogènes et biotechnologies pour la santé. 